\title{
2017 Cardiovascular and Stroke Endpoint Definitions for Clinical Trials
}

Karen A. Hicks, MD, ${ }^{\mathrm{a}, *, \uparrow}{ }_{\dagger}$ Kenneth W. Mahaffey, MD, ${ }^{\mathrm{b}}{ }_{\dagger}$ Roxana Mehran, MD, ${ }^{\mathrm{c}} \uparrow$ Steven E. Nissen, MD, ${ }^{\mathrm{d},}$ Stephen D. Wiviott, MD, ${ }^{\mathrm{e}}$ Billy Dunn, MD, ${ }^{\mathrm{f}, *}$ Scott D. Solomon, MD, ${ }^{\mathrm{g}}$ John R. Marler, MD, ${ }^{\mathrm{f}, *}$ John R. Teerlink, MD, ${ }^{\mathrm{h}}$ Andrew Farb, MD, ${ }^{\mathrm{i}, *}$ David A. Morrow, MD, MPH, ${ }^{\mathrm{e}}$ Shari L. Targum, MD, MPH, ${ }^{\mathrm{a}, *}$ Cathy A. Sila, MD, ${ }^{\mathrm{j}}$ Mary T. Thanh Hai, MD, ${ }^{\mathrm{k}, *}$ Michael R. Jaff, DO, ${ }^{1}$ Hylton V. Joffe, MD, MMSc, ${ }^{\mathrm{m}, *}$ Donald E. Cutlip, MD, ${ }^{\mathrm{n}}$ Akshay S. Desai, MD, ${ }^{\mathrm{g}}$ Eldrin F. Lewis, MD, MPH, ${ }^{\mathrm{g}}$ C. Michael Gibson, MD, MS, ${ }^{\circ}$ Martin J. Landray, $\mathrm{PHD},{ }^{\mathrm{p}}$ A. Michael Lincoff, MD, ${ }^{\mathrm{d}}$ Christopher J. White, MD, ${ }^{\mathrm{q}}$ Steven S. Brooks, MD, MBA, ${ }^{\mathrm{r}}$ Kenneth Rosenfield, MD, Michael J. Domanski, MD, ${ }^{\mathrm{t}}$ Alexandra J. Lansky, MD, ${ }^{\mathrm{u}}$ John J.V. McMurray, MD, ${ }^{\mathrm{v}}$ James E. Tcheng, MD, ${ }^{\mathrm{w}}$ Steven R. Steinhubl, MD, ${ }^{\mathrm{x}}$ Paul Burton, MD, PHD, ${ }^{\mathrm{y}}$ Laura Mauri, MD, MSc, ${ }^{\mathrm{z}}$ Christopher M. O’Connor, MD, ${ }^{\text {aa }}$ Marc A. Pfeffer, MD, PHD, ${ }^{g}$ H.M. James Hung, $\mathrm{PHD},{ }^{\mathrm{bb}, *}$ Norman L. Stockbridge, MD, PHD, ${ }^{\mathrm{a}, *}$ Bernard R. Chaitman, MD, ${ }^{\mathrm{cc}}$ Robert J. Temple, MD, ${ }^{\mathrm{dd}}$, on behalf of the Standardized Data Collection for Cardiovascular Trials Initiative (SCTI)

\section{ABSTRACT}

This publication describes uniform definitions for cardiovascular and stroke outcomes developed by the Standardized Data Collection for Cardiovascular Trials Initiative and the U.S. Food and Drug Administration (FDA). The FDA established the Standardized Data Collection for Cardiovascular Trials Initiative in 2009 to simplify the design and conduct of clinical trials intended to support marketing applications. The writing committee recognizes that these definitions may be used in other types of clinical trials and clinical care processes where appropriate. Use of these definitions at the FDA has enhanced the ability to aggregate data within and across medical product development programs, conduct metaanalyses to evaluate cardiovascular safety, integrate data from multiple trials, and compare effectiveness of drugs and devices. Further study is needed to determine whether prospective data collection using these common definitions improves the design, conduct, and interpretability of the results of clinical trials. (J Am Coll Cardiol 2018;71:1021-34) Published jointly by the American College of Cardiology Foundation and American Heart Association, Inc.

\footnotetext{
From the ${ }^{a}$ Division of Cardiovascular and Renal Products, Office of Drug Evaluation I, Center for Drug Evaluation and Research (CDER), United States Food and Drug Administration (FDA), Silver Spring, Maryland; 'Stanford Center for Clinical Research, Department of Medicine, Stanford University School of Medicine, Stanford, California; 'Division of Cardiology, Icahn School of Medicine at Mount Sinai, New York, New York; ${ }^{\mathrm{d} D e p a r t m e n t ~ o f ~ C a r d i o v a s c u l a r ~ M e d i c i n e, ~ C l e v e l a n d ~ C l i n i c, ~ C l e v e l a n d, ~ O h i o ; ~}{ }^{\mathrm{e} T I M I}$ Study Group, Cardiovascular Division, Brigham and Women's Hospital, Boston, Massachusetts; ' Division of Neurology Products, Office of Drug Evaluation I, Center for Drug Evaluation and Research (CDER), United States Food and Drug Administration (FDA), Silver Spring, Maryland; ${ }^{\mathrm{g}}$ Cardiovascular Division, Brigham and Women's Hospital, Boston, Massachusetts; ${ }^{\mathrm{h}}$ Section of Cardiology, San Francisco Veterans Affairs Medical Center and School of Medicine, University of California San Francisco, San Francisco, California; ${ }^{i}$ Division of Cardiovascular Devices, Center for Devices and Radiological Health (CDRH), United States Food and Drug Administration (FDA), Silver Spring, Maryland; ${ }^{j}$ Neurological Institute, University Hospitals-Cleveland Medical Center, Cleveland, Ohio; ${ }^{\mathrm{k} O f f i c e}$ of Drug Evaluation II, Center for Drug Evaluation and Research (CDER), United States Food and Drug Administration (FDA), Silver Spring, Maryland; 'Department of Medicine, Harvard Medical School, Boston, Massachusetts; ${ }^{\mathrm{m}}$ Division of Bone, Reproductive and Urologic Products, Office of Drug Evaluation III, Center for Drug Evaluation and Research (CDER), United States Food and Drug Administration (FDA), Silver Spring, Maryland; ${ }^{n}$ Cardiology Division, Department of Medicine, Beth Israel Deaconess Medical Center, Harvard Medical School, Boston, Massachusetts; ${ }^{\circ}$ Cardiovascular Division, Department of Medicine, Harvard Medical School, Boston, Massachusetts; ${ }^{\mathrm{P}}$ Clinical Trial Service Unit \& Epidemiological Studies Unit (CTSU), University of Oxford, Oxford, United Kingdom; ${ }^{9}$ Department of Cardiology, Ochsner Clinical School, New Orleans, Louisiana; ${ }^{\mathrm{T} B r o o k s ~ M e d t e c h, ~}$ LLC, Reisterstown, Maryland; ${ }^{\mathrm{s}}$ Vascular Medicine and Intervention, Corrigan Minehan Heart Center, Massachusetts General

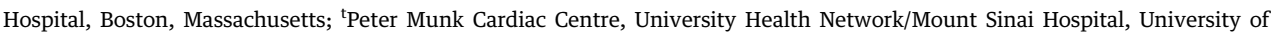




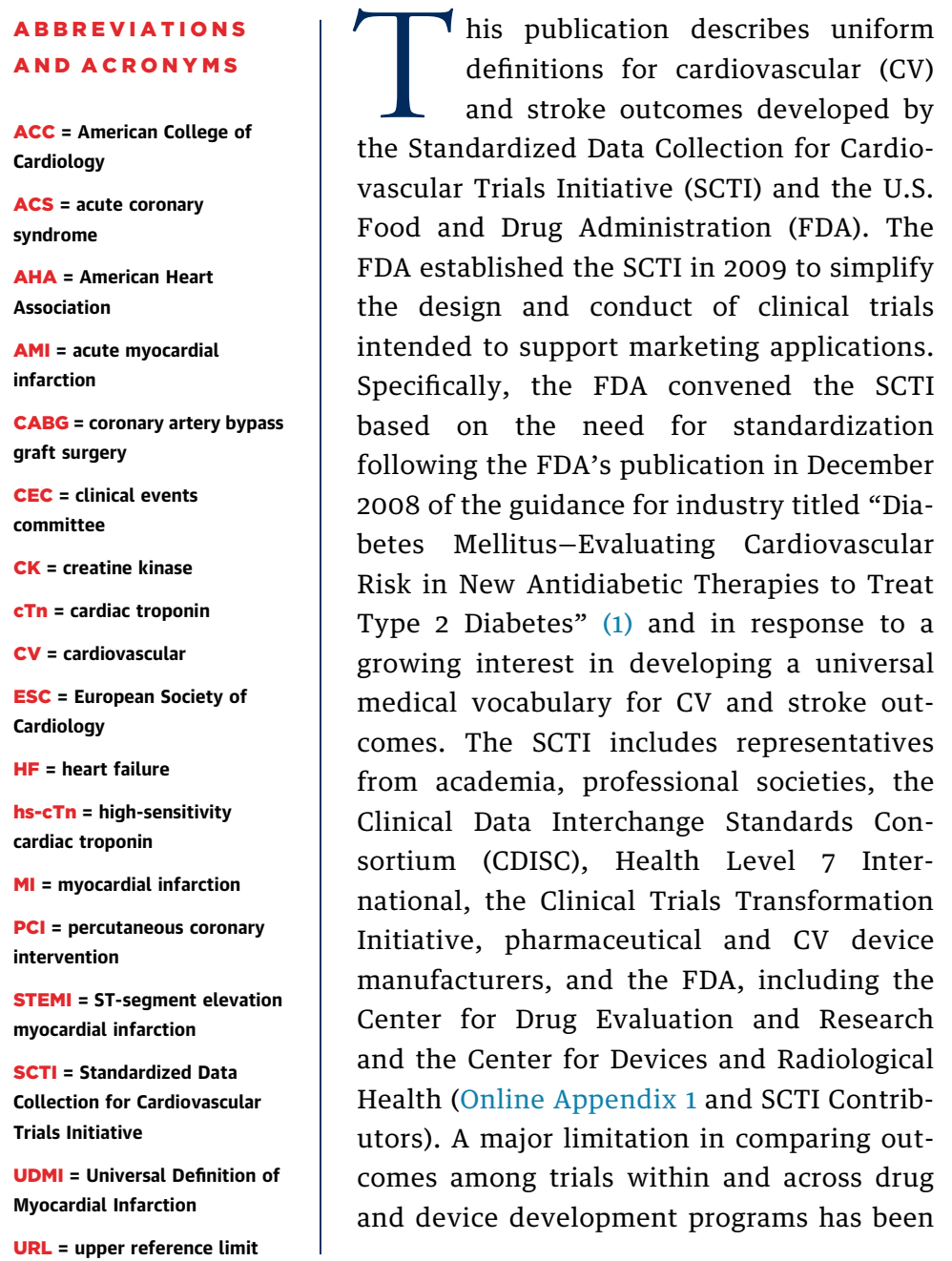

the lack of uniform definitions for key endpoint events. Pre-specified endpoint definitions that are characterized by objective criteria and reported uniformly can help address this problem. Drafts of the SCTI's CV and stroke outcome definitions have been available in the public domain since 2009 ("Standardized Definitions for Cardiovascular Outcomes Trials: Draft Recommendations," dated July 22, 2009 and discussed at the public meeting on September 11, 2009). SCTI posted these definitions for public comment on the CDISC website from November 2010 through January 2011 and on the American College of Cardiology (ACC)/American Heart Association (AHA) website from March through April of 2014 (2).

The goal of this document is to provide a framework of definitions for $\mathrm{CV}$ and stroke outcomes in clinical trials to assess the safety and effectiveness of a particular treatment (Online Appendixes 2 to 12). These definitions are based on published medical data; clinical and research expertise; published definitions and guidelines; and SCTI's interpretation of specific laboratory tests, diagnostic tests, and imaging techniques.

All definitions have limitations and will not be satisfactory in every case. Some flexibility in applying the proposed definitions may be necessary to address the specific population being studied, intervention under investigation, duration of follow-up, and disease process. Outcome measures appropriate for a particular trial may vary according to the type and objectives of the clinical trial itself, the therapeutic

Toronto, Toronto, Ontario, Canada; "Department of Internal Medicine, Section of Cardiology, Yale School of Medicine, New Haven, Connecticut; ' Institute of Cardiovascular \& Medical Sciences, BHF Cardiovascular Research Centre, University of Glasgow, Glasgow, Scotland; " Division of Cardiovascular Medicine, Duke University Medical Center, Durham, North Carolina; ${ }^{\mathrm{x}}$ Division of Digital Medicine, Scripps Translational Science Institute, La Jolla, California; ${ }^{\mathrm{y} C a r d i o v a s c u l a r}$ and Metabolism Medical Affairs,

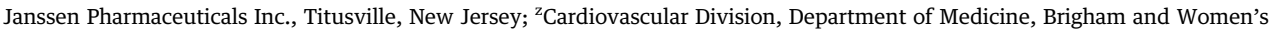

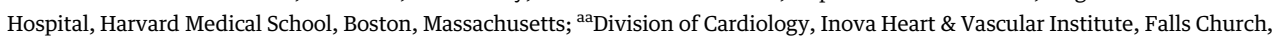
Virginia; ${ }^{b b}$ Division of Biometrics I, Office of Biostatistics, Center for Drug Evaluation and Research (CDER), United States Food and Drug Administration (FDA), Silver Spring, Maryland; ${ }^{\mathrm{cc}}$ Center for Comprehensive Cardiovascular Care, St. Louis University School of Medicine, St. Louis, Missouri; and the ${ }^{\text {dd }}$ Center for Drug Evaluation and Research (CDER), United States Food and Drug Administration (FDA), Silver Spring, Maryland. *The findings and conclusions in this report are those of the author and do not necessarily represent the official positions of the U.S. Food and Drug Administration (FDA). ${ }^{\dagger}$ Chairpersons. Dr. Mahaffey has received research grants or contracts in support of research projects from Afferent, Amgen, Apple Inc., AstraZeneca, Cardiva Medical Inc., Daiichi, Ferring, Google (Verily), Johnson \& Johnson, Luitpold, Medtronic, Merck, Novartis, Sanofi, St. Jude, and Tenax; has provided consultancy or other services for Ablynx, AstraZeneca, Boehringer Ingelheim, Bristol-Myers Squibb, Cardiometabolic Health Congress, Elsevier, GlaxoSmithKline, Johnson \& Johnson, Medergy, Medscape, Merck, Mitsubishi, Myokardia, Novartis, Oculeve, Portola, Radiometer, Springer, Theravance, UCSF, and WebMD; and has equity in BioPrint Fitness. Dr. Mehran has received personal fees for serving on the Executive Committees of Janssen Pharmaceuticals and Osprey Medical Inc.; has served on the Data and Safety Monitoring Board (DSMB) of Watermark Research Partners; has served as a consultant to Medscape, The Medicines Company, Boston Scientific, Merck, Cardiovascular Systems, Inc., Sanofi USA, Shanghai BraccoSine Pharmaceutical Corp., and AstraZeneca; has equity stock options in Claret Medical Inc. and Elixir Medical Corporation; and her institution has received research funding from Eli Lilly/Daiichi-Sankyo, Bristol-Myers Squibb, AstraZeneca, The Medicines Company, OrbusNeich, Bayer, CSL Behring, Abbott Laboratories, Watermark Research Partners, Novartis Pharmaceuticals, Medtronic, AUM Cardiovascular, Inc., and Beth Israel Deaconess Medical Center. Dr. Nissen consults for Boeringher Ingelheim, Vivus, Medtronic, Novartis, Novo Nordisk, Shire, Sanofi-Regeneron, Janssen, CiVi Biopharma, and Takeda but requires them to donate all honoraria or consulting fees directly to charity so that he receives neither income nor a tax deduction; his institution (the Cleveland Clinic Center for Clinical Research) receives funding to perform clinical trials from Abbvie, AstraZeneca, Amgen, 
area, and whether a drug or device is under study. Hence, the intent of the writing committee is not to be overly prescriptive. The writing committee recognizes that these definitions may be used in other types of trials and clinical care processes where appropriate and that definitions may change over time as new clinical information is developed or as new biomarkers or diagnostic tests become available (Online Appendix 2). The writing committee also recognizes that despite our endeavor to consolidate the best evidence and opinions to develop these definitions, studying our experience with the current definitions is needed to determine the most suitable approaches to optimize definitions for clinical outcomes.

For some large trials, questions have arisen regarding the value added by central adjudication of outcomes compared with a site-determined approach (3). In other trials, differences in event rates have been demonstrated based on approach (4). Regardless of the process used for event identification, the application of evidenced-based uniform definitions can increase the reliability and accuracy of study results.

Cerenis, Eli Lilly, Esperion, Pfizer, The Medicines Company, and Orexigen; and he is involved in these clinical trials, but receives no personal remuneration for his participation. Dr. Wiviott has received grants and personal fees from AstraZeneca, Bristol-Myers Squibb, Arena, and Eli Lilly/Daiichi-Sankyo; has received grants from Eisai, Merck, Sanofi, and Amgen; has received personal fees from Aegerion, Angelmed, Janssen, Xoma, ICON Clinical, Boston Clinical Research Institute, and Boehringer Ingelheim; and his spouse is an employee of Merck. Dr. Solomon has received research grants from Alnylam, Amgen, AstraZeneca, Bellerophon, Celladon, Gilead, GlaxoSmithKline, Ionis Pharmaceutics, Lone Star Heart, Mesoblast, MyoKardia, National Institutes of Health/ National Heart, Lung, and Blood Institute, Novartis, Sanofi Pasteur, and Theracos; and has served as a consultant for Alnylam, Amgen, AstraZeneca, Bayer, Bristol-Myers Squibb, Corvia, Gilead, GlaxoSmithKline, Ironwood, Merck, Novartis, Pfizer, Takeda, and Theracos. Dr. Teerlink has served as a consultant for Amgen, AstraZeneca, Bayer, Bristol-Myers Squibb, Celyad, Cytokinetics, Gilead, Medtronic, Merck, Novartis, Portola Pharmaceuticals, Relypsa, Stealth Biotherapeutics, St. Jude Medical, Trevena, Theravance, and ZS Pharm; and has received research support from Amgen, AstraZeneca, Bayer, Bristol-Myers Squibb, Celyad, Cytokinetics, Gilead, Medtronic, Merck, Novartis, St. Jude, and Trevena. Dr. Morrow has served as a consultant to Abbott Laboratories, Aralez Pharmaceuticals, GlaxoSmithKline, Merck, Peloton, Roche Diagnostics, and Verseon; and the TIMI Study Group has received grants from Abbott, Amgen, AstraZeneca, Daiichi-Sankyo, Eisai, GlaxoSmithKline, Johnson \& Johnson, Merck, Novartis, Pfizer, Roche Diagnostics, and Takeda. Dr. Jaff has served as a noncompensated advisor for Abbott Vascular, Boston Scientific, Cordis Corporation, and Medtronic; has served as a paid consultant for American Orthotics and Prosthetics Association, Philips/Volcano, Micell, and Venarum; has equity investment in Embolitech, Janacare, MC10, PQ Bypass, Primacea, Sano V, and Vascular Therapies; and has served as a Board Member of VIVA Physicians, a 501(c)(3) not-for-profit education and research organization. Research support has been paid to Dr. Cutlip's institution from Boston Scientific, Medtronic, and CeloNova. Dr. Desai has received consultant fees from Abbott/St. Jude Medical, Relypsa, Janssen, AstraZeneca, Merck, Sanofi, Cheetah Medical, and Novartis; and has received research support from Novartis. Dr. Lewis has received minor consultant fees from Novartis and Merck; and has received research support from Novartis, Sanofi, and Amgen. Dr. Landray's institution (University of Oxford) has received grant support from Merck, Novartis, Pfizer, Medical Research Council, British Heart Foundation, Cancer Research UK, and the National Institute for Health Research. Dr. Lincoff has served as a consultant to NovoNordisk, Amgen, and Abbott; has received significant research support from Eli Lilly; and his institution has received significant research funding from Eli Lilly, Pfizer, CSL, AstraZeneca, Esperion, and AbbVie. Dr. White has participated in the CREST2 trial (he is on the Interventional Management Committee). Dr. Brooks has received significant consulting fees from Abiomed, AUM Cardiovascular, and Ablative Solutions. Dr. McMurray's employer, Glasgow University, is currently or has recently been paid for his time as a member of the Executive or Steering Committees, Endpoint Adjudication Committees, and Data Monitoring Committees of trials testing treatments for cardiovascular disease, chronic kidney disease and diabetes, including by Abbvie, Amgen, AstraZeneca, Bristol-Myers Squibb, Cardiorentis, Dundee University, GlaxoSmithKline, Novartis, Oxford University, Merck, Resverlogix, Stealth Therapeutics, and Theracos; his employer has also been paid for meetings and presentations related to these trials and treatments; and study sponsors have paid for his travel and accommodation for some meetings related to these trials/treatments. Dr. Burton owns stock in Johnson \& Johnson; and is employed by Janssen Pharmaceuticals Inc. Dr. Mauri has served as a consultant for Amgen, Eli Lilly, St. Jude Medical, Corvia, ReCor, and Biotronik; has served as principal investigator for Recor; has received grants to her institution (all) from Amgen, Abbott, Boston Scientific, Boehringer Ingelheim, Biotronik, and Corvia; has served on the Advisory Board of St. Jude Medical; has served on the Executive Steering Committee of Corvia and Biotronik; and has received honoraria from DaiichiSankyo, AstraZeneca, and Sanofi. Dr. O’Connor has served as a consultant for ResMed, Merck, Bristol-Myers Squibb, and Actelion. Dr. Pfeffer has served as a consultant for AstraZeneca, Bayer, Boehringer Ingelheim, DalCor, Genzyme, Gilead, GlaxoSmithKline, Janssen, Lilly, The Medicines Company, Merck, Novartis, NovoNordisk, Relypsa, Sanofi, Teva, and Thrasos; has received research grant support from Novartis and Sanofi; has stock options in DalCor; and has a patent awarded to BWH regarding the use of inhibitors of the renin-angiotensin system in MI, licensed by Novartis (Dr. Pfeffer's share has been irrevocably assigned to charity). Dr. Chaitman has served on the CEC of NovoNordisk, Eli Lilly, Merck, Pharmacosmos, Janssen, Gilead, Relypsa, and DaiichiSankyo; and has served on the Data and Safety Monitoring Board of Sanofi and Tricida. All other authors have reported that they have no relationships relevant to the contents of this paper to disclose. Drs. Hicks, Mahaffey, Mehran, and Nissen served as chairpersons. Bernard J. Gersh, MB, ChB, DPhil, served as Guest Editor for this paper.

Manuscript received March 23, 2017; revised manuscript received December 21, 2017, accepted December 22, 2017. 
The following sections focus on decisions made by members of the SCTI and the rationale behind the decisions for each definition. The publication titled “2014 ACC/AHA Key Data Elements and Definitions for Cardiovascular Endpoint Events in Clinical Trials" contains the data standards for these definitions (2).

\section{DEFINITIONS}

DEATH. Death is routinely ascertained as an efficacy or safety endpoint in clinical trials (Online Appendixes 3 to 5). Although deaths from any cause may be ascertained without adjudication, assignment of the cause of death is often of scientific, clinical, and regulatory interest in both $\mathrm{CV}$ and non-CV trials. When the specific cause of death is important, adjudication using standardized definitions is recommended. In developing the definitions to categorize $\mathrm{CV}$ deaths, non-CV deaths, and deaths of undetermined cause, the Task Force aimed to distinguish the cause of death from the mode of death, and to distinguish the primary cause from intervening causes of death. The Task Force also provided definitions to subcategorize both CV deaths and non-CV deaths, but recognized challenges to the accuracy of such subcategorization. In general, such subcategorization is not needed in clinical outcomes trials (i.e., classification as $\mathrm{CV}$ or non-CV death is sufficient), but may be desired for exploratory analyses aimed at understanding disease or therapeutic mechanisms.

Classification of deaths as CV or non-CV is aimed at capturing the primary cause of death. The primary cause as defined here is the underlying disease or injury that initiated the train of events resulting in death. Thus, when an acute myocardial infarction (AMI) leads to a fatal arrhythmia, the primary cause of death would be the AMI. CV deaths include deaths that result from an AMI, sudden cardiac death, death due to heart failure (HF), death due to stroke, death due to $\mathrm{CV}$ procedures, death due to $\mathrm{CV}$ hemorrhage, and death due to other CV causes. The clinical progression toward a fatal outcome is often manifested by multiple intermediate steps, and identifying the primary cause requires careful consideration. The primary cause may be distinct from both the mode of death and an intervening cause that is temporally closer and contributes to the death.

Mode of death. The mode of death is generally regarded as the physiological derangement or the biochemical disturbance produced by the cause of death and should not be substituted for the primary cause. Non-CV causes of death (e.g., renal failure), often ultimately culminate in a CV mode of death (e.g., arrhythmia) that should not be confused with a $\mathrm{CV}$ death. In addition, overlap between the primary cause of death and mode of death can also render subclassification of $\mathrm{CV}$ deaths difficult. For example, an AMI may lead to death via a variety of modes, such as arrhythmia or myocardial rupture.

Primary versus intervening causes of death. Deaths due to primary CV causes may lead to intervening non-CV comorbidities. Intervening causes of death are other conditions that contribute to death and are part of a morbid series of events that are a result of the underlying primary cause. For example, a clinical trial subject who experiences an AMI resulting in respiratory failure due to pulmonary edema may develop a fatal infectious complication of mechanical ventilation. In this case, the infection is an intervening cause of death. Such clinical scenarios are frequent and may be interpreted variably by different reviewers applying the same standardized definition.

Subclassification of CV causes of death. For the purposes of classifying a specific CV cause of death, the proposed categories are based on the primary cause rather than the mode of death or an intervening cause such as the development of progressive HF. To support consistency in the classification of death due to AMI in particular, the definition includes deaths by any CV mechanism $\leq 30$ days after a myocardial infarction (MI) related to the immediate consequences of the MI. The period of 30 days proposed by the task force is necessarily arbitrary.

\section{Non-CV deaths and deaths of undetermined} cause. Non-CV deaths are defined as those deaths with a specific cause that is not thought to be CV in nature. Further classification of non-CV deaths is challenging because of the potential for multiple intervening causes and the need for complete knowledge of medical details that are not routinely available to a central adjudicating committee. Although not routinely necessary for outcomes trials, a suggested list of non-CV causes of death was developed by the task force if subclassification was desired. The level of detail required and the optimum classification will depend on the nature of the study population and the anticipated number and type of non-CV deaths. Therefore, this list may be adapted according to the needs of individual clinical trial investigators.

According to the definitions developed by the task force, deaths of undetermined cause should be very 
few and should be assigned only when a death cannot be attributed to a $\mathrm{CV}$ or non-CV cause due to an extreme deficiency of information (e.g., the only available information is "patient died"). Use of this category is discouraged and should be rare in wellrun clinical trials in which adequate source documentation is assiduously sought by participating investigators. A common analytic approach is to assume that all undetermined deaths are CV deaths, but the appropriate classification and analysis of undetermined causes of death depends on the population, the intervention under investigation, the duration of follow-up, and the disease process. Most importantly, the approach should be pre-specified and described in the protocol, endpoint adjudication procedures, and/or the statistical analysis plan. It should be appreciated that grouping many deaths as undetermined can create a "bias toward the null," favoring noninferiority in a noninferiority trial and a negative result in a trial seeking to show a treatment difference.

MYOCARDIAL INFARCTION. MI is an important adverse outcome used to monitor safety in drug and device development programs and efficacy in CV trials to test therapeutic strategies. The use of a standard definition for MI enhances the ability to examine incidence rates and CV safety across different clinical trials, reducing variability caused by different MI definitions (Online Appendix 6). When used by both clinicians caring for the patient and clinical events committees (CECs) that adjudicate MI events for clinical trials, a uniform definition for MI provides an opportunity to harmonize outcome reporting and focus data capture on the essential elements necessary for the diagnosis (5-8).

In 2000, a joint European Society of Cardiology (ESC)/ACC committee redefined an acute, evolving, or recent MI by specifying that any necrosis in the setting of myocardial ischemia be labeled as MI (6). The diagnosis required a typical rise and/or gradual fall of biochemical markers (cardiac troponin [cTn] preferred) of myocardial necrosis and at least 1 of the following: 1) ischemic symptoms; 2) imaging or electrocardiographic (ECG) evidence of myocardial ischemia including pathologic Q waves; 3) pathological findings of an AMI; or 4) percutaneous coronary intervention (PCI) or coronary artery bypass graft surgery (CABG) revascularization event with an increase in cardiac biomarkers. The definition was updated twice in 2007 and 2012 to identify 5 different categories of MI, discuss newer cardiac assays such as high-sensitivity cardiac troponin (hs-cTn), further differentiate myocardial injury patterns from myocardial necrosis, and revise the definition of procedure-related MI $(7,8)$. PCI-related MI is subtyped to include type 4A (periprocedural necrosis), type 4B (MI caused by stent thrombosis), and type 4C (MI caused by high-grade stenosis in a stented artery without evidence of thrombus or other explanation for the MI). The Third Universal Definition of MI (UDMI), published in 2012, requires $>5 \times$ the 99th percentile of the upper reference limit (URL) in patients with a normal cTn pre-PCI (or a rise of cTn $\geq 20 \%$ if the baseline values are elevated and stable or falling) in the first $48 \mathrm{~h}$, and in addition, requires evidence of acute ischemia/necrosis (clinical, ECG, imaging, or a flow-limiting angiographic complication). For type 5 CABG-related MI, the biomarker threshold is $>10 \times$ the 99th percentile of the URL in patients with a normal pre-procedure cTn. The Third UDMI is endorsed by the ESC/ACC/AHA and World Heart Foundation, has been adopted by the World Health Organization (WHO), and is part of the ST-segment elevation myocardial infarction (STEMI) and non-STEMI guidelines published by the ESC/ACC/ AHA. The UDMI committee acknowledges that the biomarker thresholds for types $4 \mathrm{~A}$ and $5 \mathrm{MI}$ in the 2012 definition are, in part, arbitrary and that the Society for Cardiac Angiography and Interventions and Academic Research Consortium (ARC)-2 advocate a higher biomarker threshold to diagnose significant myocardial injury/necrosis for types 4A and 5 MI (9). The SCTI plans to harmonize with thresholds specified in the Fourth UDMI, which is expected to be published in 2018.

The 2012 UDMI definition was published when high-sensitivity assays were being developed and in early use in Europe and prior to the approval of hscTn assays in the United States. In 2017, using the definition specified by the International Federation of Clinical Chemistry, the FDA approved an assay able to measure cTn values above the assay's limit of detection in $>50 \%$ of healthy individuals. Manufacturers' recommended 99th percentile URL for specific assays can be found on the International Federation of Clinical Chemistry website (10). The use of hs-cTn assays allows detection of smaller MIs previously characterized as unstable angina, thereby increasing the proportion of patients with acute coronary syndrome (ACS) classified as having had an MI. Elevated hs-cTn levels are associated with worse outcomes, including all-cause mortality $(11,12)$.

In 2017, hs-cTn assays are in widespread use worldwide and, depending on the percent of patients enrolled from countries using hs-cTn assays, may represent a sizeable proportion of all ACS events identified for adjudication. It is important to 
distinguish between myocardial injury patterns caused by acute ischemia (where a rise and/or fall in cardiac biomarkers is typically noted) and nonischemic myocardial injury patterns where cTns are often elevated, in some cases, chronically (e.g., HF, renal insufficiency, and tachycardia). If a patient has elevated cardiac biomarkers at presentation (time 0), a $\geq 20 \%$ increase in the value of the cardiac biomarkers at $3 \mathrm{~h}$ would enhance the sensitivity for the diagnosis of MI at some cost to specificity, as discussed in the following text. The larger the delta increase, the greater the likelihood of myocardial necrosis. A large delta in isolation is not diagnostic of MI and may be seen with acute myocarditis, aortic dissection, Takotsubo cardiomyopathy, pulmonary embolism, acute HF exacerbation with preserved or reduced ejection fraction, or acute renal failure. Comparisons to prior admission cTn values may be helpful in distinguishing new from chronic troponin elevation. Another approach would be to obtain a baseline cTn value prior to enrollment. Baseline cTn values performed through a core laboratory may also be helpful in clinical trials where the drug product is known to increase cTn, to stratify therapy according to baseline cTn risk, and to correlate magnitude of baseline cTn values with outcomes in subjects with abnormal pre-enrollment values.

According to the 2012 UDMI definition, "an increased cTn concentration is defined as a value exceeding the 99th percentile URL of a normal reference population" (8). The 2012 UDMI definition also indicates that "this discriminatory 99th percentile is designated as the decision level for the diagnosis of MI and must be determined for each specific assay with appropriate quality control in each laboratory" (8). The 2012 UDMI definition recommends the use of troponin assays that have high precision at lower concentration ranges, with analytical coefficient of variation $\leq 10 \%$ at the 99th percentile concentration of the URL.

For cardiac biomarkers, the 99th percentile of the URL from the respective laboratory performing the assay should be reported. Reference limits from the laboratory performing the assay are preferred over the manufacturer's listed reference limits in an assay's instructions for use. That said, the manufacturer's recommended 99th percentile of the URL for the assay is often used as the cutpoint for most hospitals, due to the expense and time required to obtain a sufficiently large and diverse population of healthy people to provide a robust estimate for the 99th percentile. The 99th percentile URL used at a local hospital may be different from (usually greater than) the manufacturer's recommended 99th percentile URL for the identical assay. The reasons a particular hospital laboratory chooses a threshold higher than the manufacturer's recommended 99th percentile URL will not be evident to a CEC. One clinical trial approach to reduce variability between sites using different thresholds for identical assays is to routinely use the manufacturer's recommended 99th percentile when evaluating a suspected MI event. An alternate, less satisfactory approach would be to use the local laboratory 99th percentile URL, recognizing that it will be difficult to know from the laboratory results whether a normal reference population was studied when the URL differs from the manufacturer's recommended value. In these cases, one should expect increased variability and heterogeneity in the diagnostic ascertainment of MI.

If cTn values are unavailable, creatine kinase MB isoform (CK-MB) measured by mass assay should be used. When CK-MB and CTn are simultaneously collected, cTn takes precedence over CK-MB because cTn has greater sensitivity and specificity. Hence, it is recommended that all hospitals adopt cTn as the preferred biomarker to diagnose MI. Measurement of total CK is not recommended for the routine diagnosis of AMI because of the wide tissue distribution of this enzyme.

When evaluating potential ACS events, CECs should be aware that different cTn assays are usually not biologically equivalent. Hence, a value of $22.5 \mathrm{ng} / \mathrm{ml}$ cTnI with assay $\mathrm{X}$ is usually not biologically equivalent to the same value obtained with assay Y. The 99th percentile value for a particular cTn assay will be dependent on the population studied (13).

A core biochemistry laboratory would obviate assay variability and could be considered in clinical trials that evaluate acute therapeutic interventions or in trials of patients with chronic cTn elevation and where the drug product is known to increase cTn values. This is not always practical in large phase 3 trials when patients are admitted to many different hospitals, and blood collection, sample preservation, personnel costs, and shipping charges for use of a core laboratory can be prohibitive.

The diagnosis of reinfarction can be a challenge after an AMI because cTn values can remain elevated for as long as 2 weeks following the index MI event and serial ECG tracings may show evolving ST$\mathrm{T}$-wave changes. Reinfarction should be considered in patients with recurrent ischemic symptoms who exhibit recurrent ST-segment deviation. For the diagnosis of myocardial infarction and reinfarction, the UDMI definition recommends an immediate troponin measurement with a second sample at either 3 to $6 \mathrm{~h}$ or earlier with newer, more sensitive cTn assays. Later samples ( $>6 \mathrm{~h}$ ) are required in patients 
with recurrent ischemia, high-risk patients, and when the timing of symptom onset is unclear. If the cTn concentration is elevated, but stable or decreasing at the time of the suspect reinfarction, $\geq 20 \%$ increase in the second cTn value is required that exceeds the 99th percentile URL. If the initial cTn concentration is normal, the criteria for new AMI apply. The magnitude of the required percent increase in cTn is somewhat arbitrary. Clearly, $a \geq 20 \%$ increase will be less convincing and specific than a $50 \%$ increase, but $\mathrm{a} \geq \mathbf{2 0} \%$ increase will be more sensitive in detecting reinfarctions. The $\geq 20 \%$ change represents a significant $(>2.77$ standard deviations of the variation associated with an elevated baseline concentration) change in cTn on the basis of a $5 \%$ to $7 \%$ analytical total coefficient of variation.

Many conditions may confound the diagnosis of MI. In a patient with known coronary disease admitted for chest pain, MI diagnosis and subtype classification can be challenging, particularly if the patient presents with hemodynamic abnormalities, raising the possibility of type 2 supply/demand MI. The diagnosis of MI can also be challenging in development programs where a particular drug product increases cTn values and the population being studied often has chronically elevated cTn values. Good clinical judgment is required based on all of the available clinical, ECG, biomarker, and imaging data. At times, missing or incomplete biomarker data and inability to time symptom onset to biomarker acquisition can confound interpretation. Hence, all of the data should be used to classify the event. For example, a hospital admission for prolonged chest pain with angiographic evidence of an ulcerated plaque or thrombus (usually accompanied by elevated biomarkers), but with no or incomplete biomarker and ECG data submitted to a CEC for adjudication could be considered sufficient to classify the MI event as a type 1 MI. A CEC should pre-specify how to classify potential MI events where there are insufficient data to make a reliable determination, and these cases will need to be accounted for in a data analysis plan.

In the AMI setting, peak cTn or CK-MB levels for a specific assay correlate with MI size and prognosis. Therefore, in clinical trials, if the sampling interval is sufficient to detect a rise and fall, it may be useful to register the peak cTn/CK-MB value and URL to allow a rough estimate of $\mathrm{MI}$ size using the ratio of biomarker/URL. Arbitrary cutpoints can be chosen to quantify small, moderate, and large MI events. Some common cutpoint ratios are $>1,>5,>10,>35,>70$, $>100$, and $>250$. Limitations of this approach are: 1 ) replicate values on the same blood specimen may not reproduce the same value depending on the biomarker assay and type; and 2) the peak value observed will depend on the frequency and timing of the sampling interval after symptom onset that may vary depending on the country and the hospitalspecific protocol for AMI management. Moreover, MI events treated with early reperfusion (i.e., revascularization or thrombolytics) or complicated by hemodynamic instability, HF, tachyarrhythmias, or life-threatening cardiac arrhythmias requiring cardioversion, might also affect the peak values.

MI can be classified as symptomatic or asymptomatic, the latter detected incidentally, or through protocol surveillance follow-up. In most clinical trials, routine (annual or more frequent) collection of ECG tracings is not advocated as an efficient mechanism to identify silent MI events (see the "Silent MI" section for further information). We believe that the proposed MI definitions are an important methodological advance for clinical trials. Standardized ascertainment and adjudication of MI endpoints should enable more accurate comparisons of MI rates in future trials, and, to the extent that cross-study comparisons are valid, allow better comparisons of MI rates among various drug and device trials. (Many limitations are inherent in cross-study comparisons; e.g., the number of MIs in a particular trial will depend on the sensitivity of the particular cTn assay used.)

Silent MI. The term silent $M I$ should apply to asymptomatic patients who develop new pathological Q-wave criteria for MI detected during routine ECG follow-up or compared with a prior visit, or who have cardiac imaging evidence of MI that cannot be directly attributed to an interim ACS event or coronary revascularization procedure. In studies where serial ECG analyses were conducted, silent Q-wave myocardial infarction (QMI) accounted for $9 \%$ to $37 \%$ of all nonfatal MI events and was associated with a significantly increased mortality risk $(14,15)$. Improper lead placement, QRS confounders, or technical error (mislabeled patient name or lead reversal) may result in what appear to be new $\mathrm{Q}$ waves or QS complexes, compared with a prior tracing. Thus, the diagnosis of a new silent QMI should be supported by a repeat ECG with correct lead placement, focused questioning about potential interim ischemic symptoms, verification that the name on the ECG corresponds to the patient in question, or an imaging study. An echocardiogram is useful if it detects wall motion abnormalities in the region of interest. However, normal wall motion does not exclude MI, and other imaging techniques such as radionuclide or cardiac magnetic resonance studies may be required to establish the diagnosis. 
There is no firm consensus on how frequently to monitor for ECG evidence of silent QMI or whether surveillance for silent MI events should be routinely implemented for particular trials.

Serial monitoring of patients who have had a symptomatic QMI event reveals Q-wave regression in a substantial number of patients (16). Q-wave regression is more common with less severe $\mathrm{Q}$-wave findings and when a single ECG territory is involved (anterior, inferior, or lateral). It may be reasonable to perform annual ECGs in clinical trials to monitor for silent QMI events if the study population is expected to have an accelerated rate of atherosclerotic events. Such patients include but are not limited to those with known or multivessel coronary artery disease (CAD) and patients with multiple atherosclerotic risk factors. When a core ECG laboratory detects a silent QMI or an investigator on routine protocol-mandated follow-up visits detects a silent MI event with ECG or imaging evidence, an MI dossier should be prepared for the CEC that includes the baseline tracing prior to randomization, interim event ECG tracings (ACS, post-coronary revascularization), protocol-mandated interval ECG tracings, and imaging evidence when available. In the review of potential silent MI events, it is recommended that the final CEC adjudication takes precedence over ECG core laboratory readings. Whether to include silent MI events in the overall assessment of MIs should be discussed with the FDA prior to study conduct.

HOSPITALIZATION FOR UNSTABLE ANGINA. Hospitalization for unstable angina (UA) is often included as an endpoint in CV clinical trials. Compared with conventional endpoints, such as death and MI, UA seems more challenging to define given the subjective assessment of the index symptoms leading to hospitalization.

Along with STEMI and non-STEMI, UA is part of the clinical spectrum of ACS. According to the National Center for Health Statistics, UA comprised $43 \%$ and $28 \%$ of the hospitalizations for primary or secondary diagnosis of ACS in 2004 and in 2010, respectively $(17,18)$. Given the higher sensitivity of current cTn assays to detect myocardial necrosis, some have questioned whether a category for UA should still exist (19), because many events previously classified as UA are now identified as MIs. The task force recognizes the need for objective criteria and defines hospitalization for UA by: 1) the character and duration of ischemic symptoms; 2) proximity of symptom onset to hospitalization; 3) duration of hospitalization; 4) at least 1 of the following: a. resting ECG evidence of acute myocardial ischemia; b. exercise or pharmacological stress testing evidence of inducible myocardial ischemia that is believed to be responsible for the ischemic symptoms/signs; c. angiographic evidence of new or worsening obstructive coronary artery disease and/or intracoronary thrombus that is believed to be responsible for the ischemic symptoms/signs; OR d. need for coronary revascularization of the presumed culprit lesion(s), as defined in c; and 5) negative cardiac biomarkers and no evidence of AMI (Online Appendix 7).

Electrocardiographic changes at the time of diagnosis are critical and include the presence or absence of ST-segment deviation and T-wave inversion, morphology of ST-segment deviation (e.g., new horizontal or downsloping ST-segment depression; new transient ST-segment elevation), and the magnitude of these changes. See Online Appendix 7 for the full criteria. Escalation of pharmacotherapy to treat ischemia may be supportive but is not diagnostic of UA.

Similar to the diagnosis of MI, many conditions may confound the diagnosis of UA. By definition, UA is cardiac biomarker negative with no evidence of AMI.

STROKE AND TRANSIENT ISCHEMIC ATTACK. Stroke is a common endpoint in CV clinical trials. Available in the public domain since 2009, the stroke definition is unique in that it combines a definition appropriate for use in clinical trials with a clinically relevant outcome measure (e.g., modified Rankin disability scale) (Online Appendix 8). Although the SCTI reviewed existing definitions for stroke and transient ischemic attack, including those published by the AHA and the National Institute of Neurological Disorders and Stroke (20-23), compared with other stroke definitions, the SCTI definition emphasizes the clinically relevant consequences of vascular brain injury to determine the safety or effectiveness of an intervention by including a measure of functional outcome (Online Appendix 8).

The information available to define stroke events varies according to trial design. For example, stroke can be a primary outcome event in a clinical trial for determining the effectiveness of a treatment to prevent stroke, or it can be an adverse safety event in trials where stroke is not the target disease. As an efficacy outcome, stroke can be the only outcome or 1 component of a composite outcome such as death, MI, and stroke. Hence, the SCTI's definition is adaptable to the many different roles that stroke 
plays as a clinical trial outcome and to the data available in the circumstances of a specific trial. Despite any differences imposed by trial design, the definition is linked to a consistent measure of effectiveness and safety across all trials.

The stroke definition minimizes technological and conceptual constraints, so that the precision of diagnosis can vary according to the relative importance of stroke in a particular trial. The unifying concept driving the definition is that stroke is a marker of potentially disabling vascular brain injury. For example, in one trial, detection of disability can require clinical assessments only when a patient reports symptoms. In other trials, there can be significant concern for even small changes in neurological function that justify the added expense of repeated brain imaging and detailed clinical stroke scales. In some trials, it can be important to know whether there is evidence of brain injury before a subject is enrolled.

Disability is a key concept in the SCTI definition. The definition is tied to a measure of disability to assess the clinically relevant consequences of vascular brain injury. The modified Rankin Scale (24) is included in the definition as an example of a suitable measure for this purpose. This simple scale would impose a minimal burden if used on all patients in all trials while providing a uniform measure of the severity of an individual stroke that could be understood across trials regardless of the population studied or the trial design. Significant brain injuries have a wide range of presentation. A uniform measure of disability makes the clinical significance of a stroke consistent across trials without specifying the use of a particular technology to assess brain injury. The focus is on the clinical significance of the presenting symptoms and any associated persistent disability as measured, for example, by the modified Rankin scale.

It is not feasible to create an algorithm to define stroke that would work for all trials and could be implemented without the exercise of clinical judgment when preparing the protocol, case report forms, and process for adjudication. Implementation of the stroke definition for a specific trial requires clinical judgment derived from a broad knowledge of the varied clinical features of stroke. The definition has flexibility that allows adaptation to the practical limits on the amount of information available for any given trial. How a specific trial implements the definition requires input from vascular neurologists and/or other experts with experience in stroke diagnosis and clinical management. If it is important, for example, to determine the stroke type, imaging to distinguish ischemic from hemorrhagic stroke may be required. A trial of an acute stroke intervention may require vascular imaging and performance of a clinical stroke scale acutely and 3 months later to assess severity and disability. The aim is for clinical trials to apply the stroke definition to assess the clinically relevant consequences of vascular brain injury.

The SCTI focused on the core definition and requirements and repeatedly resisted the temptation to generate an extensive list of desirable measures of brain injury in recognition of the wide array of trials to which the definition would be applied and the pace of innovation in the field. In an acute stroke trial, a diagnosis must be made in minutes; extensive testing comes with the cost of time, which can adversely affect the outcome under study. In stroke prevention trials, strokes may not come to an investigator's attention in time for specific tests to be performed. The treating physicians may not even realize the subject is in a trial until after the patient is discharged from the hospital. Requiring specific tests that are not universally available may lead to missing data and the failure to count significant events. A brief and reliable disability scale would remain practical in all of these situations because it can be measured after the event (e.g., 3 months) at a clinic visit with a trial investigator. This measure can also be obtained during a phone interview. In other trials, stroke may be expected to be a rare event. For these trials, serial assessments in all subjects can screen for clinically significant events that increased disability. Unexplained changes in the modified Rankin scale could precipitate a more extensive evaluation for causes that may include stroke.

The result is a definition that is broadly applicable to the wide array of trials that address 1 or more aspects of cerebrovascular disease and that maintains a uniform clinical significance of stroke outcomes across trials.

An example of an implementation of the definition is the endpoints proposed independently by the NeuroARC group for neurological outcomes focusing primarily on CV interventions (25). Like NeuroARC, we agree that standardized approaches for ascertainment and definition of neurological endpoints in clinical trials are important. The SCTI definitions were created to allow more generalized use across all clinical trials evaluating $\mathrm{CV}$ endpoints and do not require a particular imaging modality to define stroke. Hence, the SCTI definitions are more suitable for implementation in global clinical trials, where sites may not have access to advanced imaging techniques. In addition, the SCTI definitions allow the use of symptom persistence to distinguish transient 
ischemia from acute infarction, but leave the specific definition of persistence to stroke experts participating in the design of each trial. In summary, the SCTI definitions are more applicable to a wide variety of clinical trial designs that have stroke or TIA endpoints while maintaining a consistent assessment of the clinical significance of these events across trials.

HF EVENT. Hospitalized and nonhospitalized HF events are relevant endpoints in HF trials and trials of non-HF therapies in which the therapy might affect the risk of HF. An HF event includes hospitalizations for $\mathrm{HF}$ and urgent outpatient visits, and is defined as a constellation of signs, symptoms, diagnostic testing, and HF-directed therapy, as described in the following text (Online Appendix 9). For the purpose of study reporting, HF hospitalizations and urgent HF visits should be collected and reported separately.

HF hospitalization. An HF hospitalization has been associated with an increased subsequent risk of mortality (26), and this endpoint has been a relevant discriminator of tested therapies in clinical trials. To fulfill the criteria for an HF hospitalization, a patient is required to have an unscheduled hospital admission for a primary diagnosis of $\mathrm{HF}$ with a length of stay that either exceeds $24 \mathrm{~h}$ or crosses a calendar day (if hospital admission and discharge times are unavailable). The patient is also required to have typical signs, symptoms, and diagnostic testing results consistent with the diagnosis of HF (27). Laboratory findings consistent with $\mathrm{HF}$ include elevated natriuretic peptides, radiological evidence of congestion, and either echocardiographic or invasive evidence of elevated filling pressures. In addition to these signs and symptoms, the patient should also receive treatment specifically directed at $\mathrm{HF}$, including at least 1 of the following: 1 ) significant augmentation in oral diuretic therapy; 2) initiation of intravenous diuretic (even a single dose) or vasoactive agent (e.g., vasodilator, vasopressor, or inotropic therapy); or 3) mechanical circulatory support or fluid removal. Significant augmentation of oral diuretic therapy is defined, for example, as the doubling of loop diuretic dose; initiation of maintenance loop diuretic therapy; or initiation of combination diuretic therapy to relieve congestion. Combination diuretic therapy could include: 1) a thiazide-type diuretic (e.g., hydrochlorothiazide, metolazone, or chlorothiazide) plus a loop diuretic; or 2) a mineralocorticoid receptor antagonist (e.g., spironolactone or eplerenone) plus a loop diuretic. Mechanical fluid removal includes ultrafiltration, hemofiltration, and dialysis as well as thoracentesis or paracentesis for HF management.
Each criterion of the HF hospitalization definition has been associated with increased risk.

Urgent outpatient visits. To satisfy the criteria for a nonhospitalized HF event, the patient must have an urgent, unscheduled office or emergency visit for HF with signs, symptoms, and diagnostic testing results identical to those already described for an HF hospitalization. The patient must also (with the exception of significant augmentation in oral diuretic therapy) require therapy similar to that previously described for an HF hospitalization. It is important to note that clinic visits for scheduled administration of $\mathrm{HF}$ therapies or procedures (e.g., intravenous diuretics, intravenous vasoactive agents, or mechanical fluid removal) do not qualify as nonhospitalized HF events.

The inclusion of these events in the definition will ensure optimal capture of HF events despite differences in the threshold for hospitalization worldwide and increasing pressure, especially in the United States, to reduce the number of HF hospitalizations. $\mathrm{HF}$ events that are not hospitalizations, if based on strict criteria, have prognostic significance similar to HF hospitalizations $(28,29)$.

PERCUTANEOUS CORONARY INTERVENTION. Catheterbased cardiac procedures remain the cornerstone of diagnostic and therapeutic modalities for many patients with CAD. Although these procedures are primarily focused on coronary anatomic characteristics, invasive coronary imaging is still considered a gold standard on which the sensitivity and specificity of noninvasive diagnostic tests rely. The tools used in diagnostic invasive coronary procedures include angiography, intravascular ultrasound, and optical coherence tomography. Fractional flow reserve is increasingly utilized to assess the functional severity of anatomically intermediate coronary artery lesions to guide clinically appropriate revascularization.

PCI procedures can have mechanistic and clinical objectives. The primary mechanistic objective is to enhance myocardial blood flow by increasing coronary luminal area in significantly stenotic lesions. The clinical goals of PCI are most often targeted toward relieving (or improving) symptoms in patients with angina or improving survival and preserving left ventricular function (and reducing the risk of $\mathrm{HF}$ ) in patients with ACS. Therapeutic devices used in interventional cardiology procedures include percutaneous transluminal coronary angioplasty balloons, cutting/scoring balloons, atherectomy devices, baremetal stents, metallic drug-eluting stents (DES) with durable or biodegradable polymer coatings, and fully bioabsorbable drug-eluting scaffolds. Each of these 


\section{CENTRAL ILLUSTRATION Pathway to Successful Regulatory Submissions}

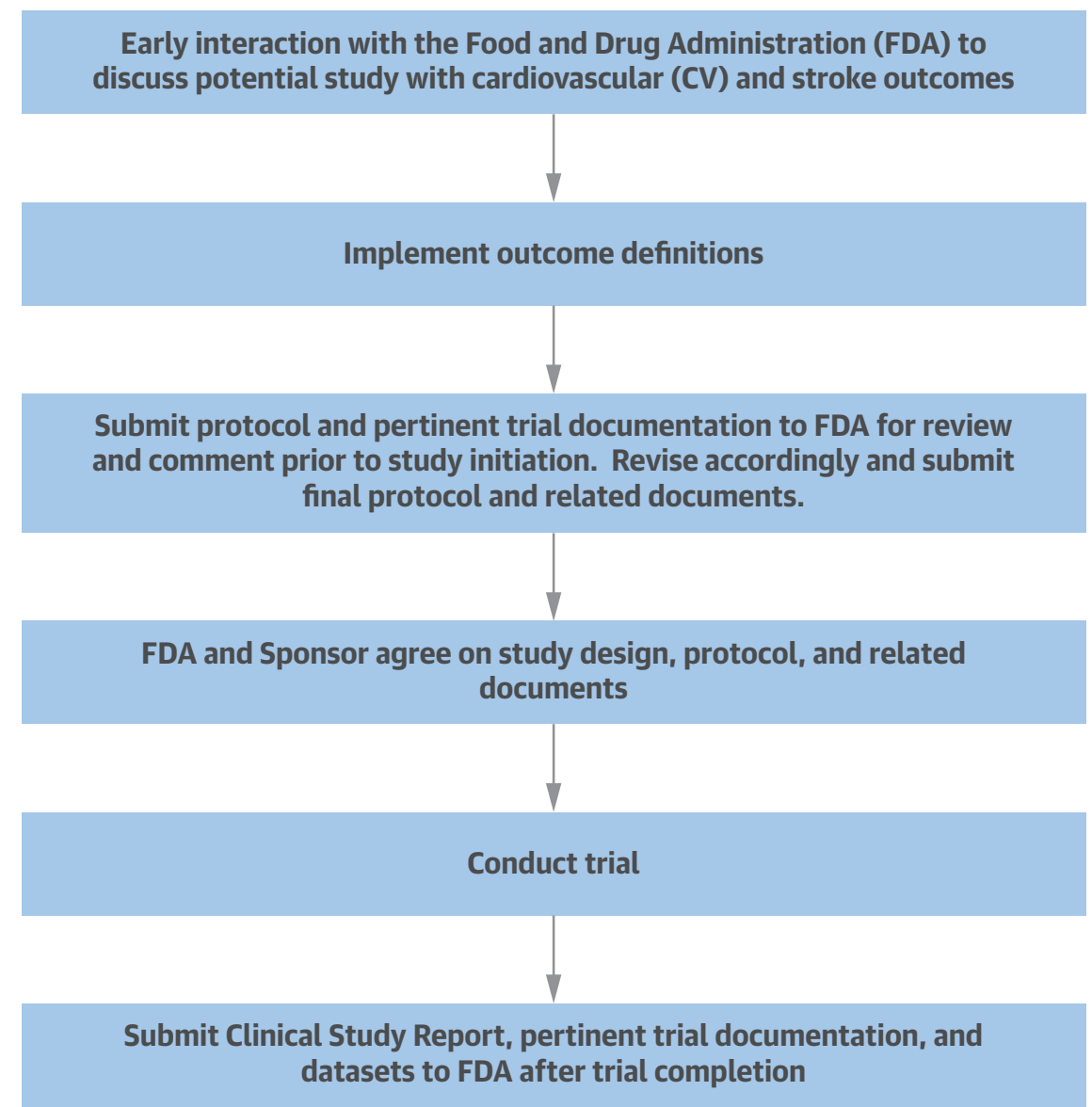

Hicks, K.A. et al. J Am Coll Cardiol. 2018;71(9):1021-34.

Pathway for potential studies with cardiovascular (CV) and stroke outcomes. FDA = U.S. Food and Drug Administration.

interventional cardiology device types has its own expected rates of procedural and long-term performance. Over the course of the past 3 decades, device innovation has progressed to address the limitations of prior technologies: 1) bare-metal stents were developed to prevent acute recoil and abrupt vessel closure as well as negative arterial remodeling associated with percutaneous transluminal coronary angioplasty; 2) DES reduce repeat revascularization rates by inhibiting neointimal proliferation that occurs within bare-metal stents; 3) DES with biodegradable polymer coatings were produced with the hope of reducing the risk of late adverse vascular responses to permanent polymers; and 4) it is hoped that fully bioabsorbable drug-eluting scaffolds may restore normal arterial vasomotion and prevent very late restenosis associated with the presence of a permanent metallic stent. Further, substantial advances in procedural techniques and in device design have allowed operators to treat patients with increasingly complex clinical presentations and coronary lesion morphologies.

In clinical trials enrolling patients with established CAD or CV risk factors, it is expected that many patients will have coronary events or symptoms suggestive of coronary insufficiency during the course of the study. Many of these patients will undergo invasive coronary artery assessments and catheter-based interventions. Because of the heterogeneity of clinical presentations and the types of coronary interventions, a common set of interventional cardiology clinical and anatomic event definitions provide a uniform language and approach for clinical study data capture. Importantly, ascertainment of specific data elements depends on the type and objectives of the clinical trial itself. For 
- Cardiovascular (CV) and stroke outcomes are important in CV and non-CV trials

- Lack of uniform event definitions compromises the ability to compare CV and stroke outcomes among trials within and across drug and device development programs

- The proposed definitions have been developed by a comprehensive process

- Key stakeholders include the public, academia, industry, and the United States Food and Drug Administration

- The proposed definitions will facilitate the systematic and uniform characterization of CV and stroke outcomes

- Further study is needed to determine whether prospective data collection using these common definitions improves the design, conduct, and interpretability of the results of clinical trials

- Refinement of these definitions may be necessary as we learn more about the diagnosis and prognosis of these CV and stroke outcomes

example, a study examining 2 coronary revascularization strategies (e.g., PCI vs. CABG or an investigational DES vs. an approved DES) would be expected to collect highly detailed coronary artery anatomic and revascularization procedural data to assess the comparative safety and effectiveness of the treatment groups. Conversely, much less granular anatomic and revascularization procedural information would be needed in a trial of an investigational diabetes or $\mathrm{HF}$ drug in patients with established CAD.

The interventional cardiology definitions presented in Online Appendix 10 are primarily relevant to PCI trials. These definitions include a description of coronary arterial segments, coronary flow grades, and selected adverse events (mostly associated with revascularization procedures such as abrupt vessel closure, arterial dissection, and restenosis). The definitions distinguish among target lesions, target vessels, and nontarget lesions/vessels, and some include anatomic and clinical features. For example, clinically driven target lesion revascularization, the most common primary endpoint in trials to assess the safety and effectiveness of coronary stents, includes a lesion severity metric (a target lesion diameter stenosis $>70 \%$ ) and evidence of clinical or physiological ischemia. Composite endpoints that have been useful in coronary revascularization studies, such as target lesion/vessel failure, are presented; these composites combine safety (cardiac death and MI related to the target vessel) and effectiveness (ischemia-driven revascularization of the target lesion/vessel) elements. Finally, the list provides definitions of specific access-site complications of interest.

PERIPHERAL VASCULAR INTERVENTION. With the advances in technology now emerging for patients with noncardiac peripheral vascular diseases, the definitions described here are designed to uniformly capture outcomes in clinical trials (Online Appendix 11). Given that the preponderance of trials is in the peripheral arterial vascular bed, we have chosen to concentrate endpoint definitions on extracranial and peripheral arterial trials, and have not attempted to define venous intervention trials. Endpoint definitions related to carotid/cerebral revascularization, peripheral arterial surgical revascularization, and treatment of aortic diseases are also beyond the scope of this publication.

The definitions in this section highlight both the resolution of the hemodynamically significant arterial obstruction while ameliorating symptoms prompting the intervention, particularly in the lower extremity arteries. In the lower extremity arteries, there are different clinical syndromes, often based on the timing of onset and severity of symptoms. We recognize the importance of the timing and type of intervention based on the acuity of the symptoms, thereby defining elective, urgent, and emergent cases.

Anatomically, we attempted to highlight the differences between the "culprit" lesion resulting in the need for intervention as opposed to the artery in which the target lesion exists. This difference 
between target lesion and target vessel is important in comparing the effectiveness of different devices for the treatment of the same arterial segment.

Finally, we highlight the importance of independent adjudication of anatomic outcomes in follow-up of devices used in peripheral artery interventions.

STENT THROMBosis. According to the classification proposed by the ARC (30), the definition of stent thrombosis relies on: 1) the timing of the event; and 2) likelihood of thrombosis as the proximate cause (Online Appendix 12). The time interval for defining stent thrombosis begins after successful completion of the procedure. Stent thrombosis can be reported as a cumulative value over time or within the specific time periods as follows: 1 ) early (o to 30 days); 2) late (31 days to 1 year); and 3) very late ( $>1$ year). Early stent thrombosis also includes acute (o to $24 \mathrm{~h}$ ) and subacute ( 1 to 30 days) intervals. Stent thrombosis is defined as definite, probable, or possible. Definite stent thrombosis is defined as occurring in the setting of ACS when there is confirmation of stent occlusion or thrombus by angiography or autopsy examination. Probable stent thrombosis is defined as death occurring within 30 days that cannot be attributed to another cause, or when MI occurs at any time point and is attributable to the target vessel without angiographic confirmation of another culprit lesion. Possible stent thrombosis is defined as death occurring later than 30 days that cannot be attributed to another cause. Classification of these events requires the following key information: clinical details surrounding the acute event; dates and procedural information for all prior stent placement procedures; serial ECGs at the time of the event and, for appropriate duration of follow-up, serial cardiac biomarkers confirming MI; clinical details surrounding all deaths, including death certificate and autopsy report; and review of coronary angiograms by either an independent angiographic core laboratory or independent CEC. When available data satisfy the criteria for more than 1 classification, the highest level of certainty should be reported.

\section{SUMMARY AND CONCLUSIONS}

This publication is the product of a collaboration among academia, clinical experts, industry, and the FDA. The aim of this collaboration is to develop uniform definitions for $\mathrm{CV}$ and stroke outcomes and to simplify the design and conduct of clinical trials intended to support marketing applications (Central Illustration, Figure 1).

Since the inception of the SCTI in 2009, use of these definitions at the FDA has enhanced the ability to aggregate data within and across medical product development programs, conduct meta-analyses to evaluate CV safety, integrate data from multiple trials, and compare effectiveness of drugs and devices.

Further study is needed to determine whether prospective data collection using these common definitions improves the design, conduct, and interpretability of the results of clinical trials.

ADDRESS FOR CORRESPONDENCE: Dr. Karen A. Hicks, Division of Cardiovascular and Renal Products, Office of Drug Evaluation I, Center for Drug Evaluation and Research (CDER), U.S. Food and Drug Administration, 10903 New Hampshire Avenue, Building 22, Room 4182, Silver Spring, Maryland 20993-0002. E-mail: karen.hicks@fda.hhs.gov.

\section{REFERENCES}

1. U.S. Food and Drug Administration. Center for Drug Evaluation and Research. Guidance for industry: diabetes mellitus-evaluating cardiovascular risk in new antidiabetic therapies to treat type 2 diabetes. December 2008. Available at: https://www.fda.gov/downloads/Drugs/Guidance ComplianceRegulatorylnformation/Guidances/ucm 071627.pdf. Accessed January 4, 2018.

2. Hicks KA, Tcheng JE, Bozkurt B, et al. 2014 ACC/AHA key data elements and definitions for cardiovascular endpoint events in clinical trials: a report of the American College of Cardiology/ American Heart Association Task Force on Clinical Data Standards (Writing Committee to Develop Cardiovascular Endpoints Data Standards). J Am Coll Cardiol 2015;66:40-69.

3. Granger $\mathrm{CB}$, Vogel V, Cummings SR, et al. Do we need to adjudicate major clinical events? Clin Trials 2008;5:56-60.
4. Mahaffey KW, Harrington RA, Akkerhuis $M$, et al. Disagreements between central clinical events committee and site investigator assessments of myocardial infarction endpoints in an international clinical trial: review of the PURSUIT study. Curr Control Trials Cardiovasc Med 2001;2: 187-94.

5. Nomenclature and Criteria for Diagnosis of Ischemic Heart Disease. Report of the Joint International Society and Federation of Cardiology/ World Health Organization Task Force on Standardization of Clinical Nomenclature. Circulation 1979;59:607-9.

6. Alpert JS, Thygesen $K$, Antman $E$, et al. Myocardial infarction redefined: a consensus document of the Joint European Society of Cardiology/American College of Cardiology Committee for the Redefinition of Myocardial Infarction. J Am Coll Cardiol 2000;36:959-69.
7. Thygesen $\mathrm{K}$, Alpert JS, White HD, on behalf of the Joint ESC/ACCF/AHA/WHF Task Force for the Redefinition of Myocardial Infarction. Universal definition of myocardial infarction. J Am Coll Cardiol 2007;50:2173-95.

8. Thygesen $K$, Alpert JS, Jaffe AS, Simoons ML, Chaitman BR, White $H D$, on behalf of the Joint ESC/ACCF/AHA/WHF Task Force for the Universal Definition of Myocardial Infarction. Third universal definition of myocardial infarction. J Am Coll Cardiol 2012; 60:1581-98.

9. Moussa ID, Klein LW, Shah B, et al. Consideration of a new definition of clinically relevant myocardial infarction after coronary revascularization. An expert consensus document from the Society for Cardiovascular Angiography and Interventions (SCAI). J Am Coll Cardiol 2013;62: 1563-70. 
10. International Federation of Clinical Chemistry and Laboratory Medicine. Available at: www.ifcc.org. Accessed January 11, 2018.

11. Keller $T$, Zeller $T$, Ojeda $F$, et al. Serial changes in highly sensitive troponin I assay and early diagnosis of myocardial infarction. JAMA 2011;306:2684-93.

12. Melki D, Lugnegård J, Alfredsson J, Lind $S$ Eggers KM, Lindahl B, Jernberg T. Implications of introducing high-sensitivity cardiac troponin into clinical practice. Data from the SWEDEHEART Registry. J Am Coll Cardiol 2015;65:1655-64.

13. Gore $M O$, Seliger $S L$, deFilippi $C R$, et al. Ageand sex-dependent upper reference limits for the high-sensitivity cardiac troponin T assay. J Am Coll Cardiol 2014;63:1441-8.

14. Chaitman BR, Hardison RM, Adler $D$, et al. Bypass Angioplasty Revascularization Investigation 2 Diabetes (BARI 2D) Study Group. The bypass angioplasty revascularization investigation 2 diabetes randomized trial of different treatment strategies in type 2 diabetes mellitus with stable ischemic heart disease: Impact of treatment strategy on cardiac mortality and myocardial infarction. Circulation 2009:120:2529-40.

15. Burgess DC, Hunt $D$, Li L, et al. Incidence and predictors of silent myocardial infarction in type 2 diabete and the effect of fenofibrate: an analysis from the Fenofibrate Intervention and Event Lowering in Diabetes (FIELD) study. Eur Heart J 2010;31:92-9.

16. Karnegis JN, Matts J, Tuna $\mathrm{N}$, for the POSCH Group. Development and evolution of electrocardiographic Minnesota Q-QS codes in patients with acute myocardial infarction. Am Heart J 1985;110:452-9.

17. Thom $T$, Haase $N$, Rosamond $W$, et al. Hear disease and stroke statistics-2006 update: a report from the American Heart Association Statistics Committee and Stroke Statistics Subcommittee. Circulation 2006;113:e85-151.

18. Mozaffarian D, Benjamin EJ, Go AS, et al. Hear disease and stroke statistics-2016 update. a report from the American Heart Association. Circulation 2016:132:e1-324.

19. Braunwald $E$, Morrow DA. Unstable angina: is it time for a requiem? Circulation 2013;127:2452-7.

20. Easton JD, Saver JL, Albers GW, et al. Definition and evaluation of transient ischemic attack: scientific statement for healthcare professionals from the American Heart Association/American Stroke Association Stroke Councili Council on Cardiovascular Surgery and Anesthesia; Council on Cardiovascular Radiology and Intervention; Council on Cardiovascular Nursing; and the Interdisciplinary Council on Peripheral Vascular Disease. Stroke 2009;40:2276-93.

21. Sacco RL, Kasner SE, Broderick JP, et al. An updated definition of stroke for the 21st century: statement for healthcare professionals from the American Heart Association/American Stroke Association. Stroke 2013;44:2064-89.

22. National Institute of Neurological Disorders and Stroke. Classification of cerebrovascular diseases III. Stroke 1990;21:637-76.

23. Albers GW, Caplan LR, Easton JD, et al. Transient ischemic attack-proposal for a new definition. N Engl J Med 2002;347:1713-6.

24. Van Swieten JC, Koudstaal PJ, Visser $M C$ Schouten HJA, van Gijn J. Interobserver agree ment for the assessment of handicap in stroke patients. Stroke 1988;19:604-7.

25. Lansky AJ, Messé SR, Brickman AM, et al. Proposed standardized neurological endpoints fo cardiovascular clinical trials. An Academic Research Consortium initiative. J Am Coll Cardiol 2017;69:679-91.

26. Solomon SD, Dobson J, Pocock $S$, et al., for the Candesartan in Heart failure: Assessment of Reduction in Mortality and morbidity (CHARM) Investigators. Influence of nonfatal hospitalization for heart failure on subsequent mortality in patients with chronic heart failure. Circulation 2007:116:1482-7.

27. McKee PA, CastelliWP, McNamara PM, Kannel WB. The natural history of congestive heart failure: the Framingham study. N Engl J Med 1971;285:1441-6.

28. Skali $H$, Dwyer EM, Goldstein $R$, et al. Prognosis and response to therapy of first inpatient and outpatient heart failure event in a heart failure clinical trial: MADIT-CRT. Eur J Heart Fail 2014;16:560-5.

29. Okumura N, Jhund PS, Gong J, et al., for the PARADIGM-HF Investigators and Committees. Importance of clinical worsening of heart failure treated in the outpatient setting: evidence from the prospective comparison of ARNI with ACEI to determine impact on global mortality and morbidity in heart failure trial (PARADIGM-HF). Circulation 2016;133:2254-62

30. Cutlip DE, Windecker $S$, Mahran $R$, et al., on behalf of the Academic Research Consortium. Clinical endpoints in coronary stent trials: a case for standardized definitions. Circulation 2007;115: 2344-51.

KEY WORDS cardiovascular and strok endpoint definitions, cardiovascular and stroke outcome definitions, cardiovascular and stroke outcomes, cardiovascular endpoint definitions, cardiovascular endpoints, cardiovascular outcomes, clinical trial endpoint definitions, clinical trial endpoints, endpoint definitions, endpoints, outcome definitions, stroke endpoint definitions, stroke endpoints, stroke outcomes

APPENDIX For a full list of authors and contributors as well as expanded definition criteria, please see the online version of this paper. 